\title{
TIP, A Novel Host Factor Linking Callose Degradation with the Cell-to-Cell Movement of Potato virus $X$
}

\author{
Ingela Fridborg, ${ }^{1}$ Jef Grainger, ${ }^{1}$ Anthony Page, ${ }^{1}$ Mark Coleman, ${ }^{2}$ Kim Findlay, ${ }^{3}$ and Susan Angell ${ }^{1}$ \\ ${ }^{1}$ Department of Disease and Stress Biology, John Innes Centre, Norwich, NR4 7UH, U.K.; ${ }^{2}$ School of Biological Sciences, \\ University of East Anglia, Norwich, U.K.; ${ }^{3}$ Department of Cell and Developmental Biology, John Innes Centre, Norwich, \\ NR4 7UH, U.K.
}

Submitted 13 June 2002. Accepted 29 October 2002.

\begin{abstract}
The cell-to-cell movement of Potato virus $X(\mathrm{PVX})$ requires four virus-encoded proteins, the triple gene block (TGB) proteins (TGB25K, TGB12K, and TGB8K) and the coat protein. TGB12K increases the plasmodesmal size exclusion limit (SEL) and may, therefore, interact directly with components of the cell wall or with plant proteins associated with bringing about this change. A yeast two-hybrid screen using TGB12K as bait identified three TGB12K-interacting proteins (TIP1, TIP2, and TIP3). All three TIPs interacted specifically with TGB12K but not with TGB25K or TGB8K. Similarly, all three TIPs interacted with $\beta-1,3-$ glucanase, the enzyme that may regulate plasmodesmal SEL through callose degradation. Sequence analyses revealed that the TIPs encode very similar proteins and that TIP1 corresponds to the tobacco ankyrin repeat-containing protein HBP1. A TIP1::GFP fusion protein localized to the cytoplasm. Coexpression of this fusion protein with TGB12K induced cellular changes manifested as deposits of additional cytoplasm at the cell periphery. This work reports a direct link between a viral movement protein required to increase plasmodesmal SEL and a host factor that has been implicated as a key regulator of plasmodesmal SEL. We propose that the TIPs are susceptibility factors that modulate the plasmodesmal SEL.
\end{abstract}

Additional keywords: plasmodesmata, virus movement.

The cell-to-cell movement of plant viruses is a complex process involving a variety of virus-encoded proteins and host factors. Plant viruses move from cell to cell via plasmodesmata, the cytoplasmic connections that link cells (Carrington et al. 1996; Lucas and Gilbertson 1994). Each plasmodesma is lined by a plasma membrane and consists of a cytoplasmic annulus divided into microchannels. These channels are formed by "linking proteins" and are associated with the appressed membranes of the endoplasmic reticulum (the desmotubule) (Overall and Blackman 1996; Zambryski and Crawford 2000). At the neck region, there is a constriction of the plasmodesmal canal. It is thought that the size exclusion limit (SEL) is regulated in this region through callose metabolism, possibly modulated by (1-3)- $\beta$-D-glucan synthase and class I $\beta-1,3-$ glucanase (Botha and Cross 2000; Northcote et al. 1989). The

Corresponding author: S. Angell; Telephone: +44 1603 450235; Fax: +44 1603 450045; E-mail: sue.angell@bbsrc.ac.uk.

Current address of I. Fridborg: Department of Plant Biology, Swedish University of Agricultural Sciences, Box 7080, SE-750 07, Uppsala, Sweden plasmodesmal SEL is considerably smaller than the molecular size of viruses. Thus, the cell-to-cell trafficking of viruses is often associated with a change in the SEL (Angell et al. 1996; Derrick et al. 1992; Fujiwara et al. 1993; Vaquero et al. 1994; Wolf et al. 1989). The virus-encoded proteins that mediate this plasmodesmal "gating" are usually termed movement proteins (MPs).

The process of cell-to-cell movement is not yet fully understood but involves intracellular trafficking of the virus from its site of replication to the cell wall followed by modification of plasmodesmata to permit movement of the viral nucleic acid or viral particle through these conduits. The targeting to and movement of the virus through plasmodesmata requires several interactions involving the virus, MPs, and cellular components.

Potato virus $X$ (PVX) is the type member of the Potexvirus group and, like Hordei-, Carla-, Beny-, and Furoviruses, requires the triple gene block (TGB) proteins to move between cells. The PVX TGB open reading frames are partially overlapping and encode the TGB25K, TGB12K, and TGB8K proteins. Cell-to-cell movement of PVX also requires the coat protein (CP) (Chapman et al. 1992; Fedorkin et al. 2001).

The TGB proteins are likely to form many interactions with cellular components (and with CP [Oparka et al. 1996; Santa Cruz et al. 1998]) to facilitate PVX movement, but the mechanisms by which these proteins mediate cell-to-cell movement are largely unknown. However, several studies have identified specific functions for each TGB protein. For example, mutations in the PVX TGB25K or TGB12K proteins abolish viral movement (Chapman et al. 1992; Fedorkin et al. 2001), whereas mutation of TGB8K only delays movement (Tamai and Meshi 2001). Therefore, TGB8K is not absolutely required for cell-to-cell movement. Microinjection studies have indicated that the cell-to-cell movement of PVX involves a change in the plasmodesmal SEL (Angell et al. 1996). TGB25K may be involved in mediating this change (Angell et al. 1996), but TGB12K is absolutely required (Tamai and Meshi 2001). TGB8K does not increase the SEL (Tamai and Meshi 2001). TGB25K can prevent systemic gene silencing (Voinnet et al. 2000) and can move between cells in the absence of the other two TGB proteins (Yang et al. 2000). Solovyev and associates (2000) have suggested that TGB12K and TGB8K transport viral RNAs within the cell along endomembranes and that the subcellular location of TGB12K may be altered in the presence of TGB8K. Tamai and Meshi (2001) propose that TGB25K and TGB12K can move between cells, whereas TGB8K may only function in those cells expressing the protein.

The ability of TGB12K to modify the plasmodesmal SEL suggests that this protein may interact directly with one or more components of the plasmodesmata to bring about this 
change. Alternatively, the binding of TGB12K to a host factor may activate a response at the cell periphery that results in modification to plasmodesmata. An infection will only succeed if the virus can complete this series of reactions with host proteins and cellular structures. The molecular mechanism by which this viral MP mediates a change in the SEL of plasmodesmata is still largely unknown.

To gain a better understanding of how TGB12K mediates a change in the SEL of plasmodesmata, we searched for host factors that are involved in this viral MP-mediated cellular modification. We used an approach that would identify direct protein-protein interactions between TGB $12 \mathrm{~K}$ and host factors. We identified three candidates, termed TIPs for TGB12K-interacting proteins. We show that all three TIPs can also interact with $\beta$-1,3-glucanase, thus providing the link between a viral MP required for plasmodesmal gating and a host protein that has been proposed to regulate the plasmodesmal SEL. The identity of these host factors and their ability to form proteinprotein interactions allow us to speculate as to the molecular mechanism by which PVX might promote its cell-to-cell movement through modification of plasmodesmal SEL.

\section{RESULTS}

\section{Isolation of TGB12K-interacting host factors.}

To identify possible protein-protein interactions between TGB12K and plant proteins, we used the LexA-based yeast two-hybrid system. A cDNA library was generated from noninfected Nicotiana tabacum cv. Samsun NN leaves and introduced into yeast cells. Using TGB12K as the target (bait), we screened approximately $10^{6} \mathrm{cDNA}$ clones and identified more than 100 cDNAs encoding proteins that interacted with TGB12K. A positive interaction was scored as growth plus blue coloration on assay medium (SD/Gal/Raf/-His/-Leu/-Trp/Ura/X-gal, discussed below).

TGB12K-interacting clones (23) were selected for sequence analyses. One cDNA encoded aldehyde dehydrogenase; this clone is not further described here. The remaining 22 cDNAs encoded one of three proteins. We have termed these proteins TIP1, TIP2, and TIP3. From these 22 cDNAs, TIP1, TIP2, and TIP3 were identified seven, nine, and six times, respectively. Neither TIP1, TIP2, TIP3, nor aldehyde dehydrogenase were identified in an additional library screen using TGB8K as bait (S. Angell, unpublished data). Similarly, no protein-protein interaction was observed in yeast expressing each TIP with either TGB8K, TGB25K, or three unrelated plant proteins as controls (ran, parf2, and protochlorophyllide reductase) (Fig. 1; Table 1), indicating that the interactions were specific to TGB12K.

Multiple sequence alignments indicated that the TIPs are very similar proteins (Fig. 2A). To check the fidelity of the cDNA library and to rule out the possibility that the three TIP sequences were variants of the same cDNA generated during library amplification, we assessed whether all three TIPs were expressed in plants. We performed reverse transcription-polymerase chain reaction (RT-PCR) on noninfected $N$. tabacum cv. Samsun NN total RNA, using TIP-specific primers. The primers were designed to two regions in which the nucleotide sequence is identical between TIP1, TIP2, and TIP3 but which are separated by a stretch of nonidentical sequence, such that all three TIP sequences would be amplified if expressed in noninfected plants. Sequence analysis of 20 cloned PCR products revealed two distinct products specifically correlating to the TIP1 and TIP2 sequences (data not shown). The TIP3 sequence was not identified among these 20 clones.

To confirm the protein-protein interactions between TGB12K and the TIPs, we performed a far-Western analysis with TIP1. We expressed the TIP1 sequence in E. coli strain BL21 and TGB12K tagged with the HA peptide sequence (YPYDVPDYA) derived from the human influenza hemagglutinin protein in BL21. Membranes were generated by transfer of crude protein extracts from BL21 expressing TIP1 separated by sodium dodecyl sulfate-polyacrylamide gel electrophoresis. These membranes were probed with crude cell extracts of BL21 expressing TGB12K-HA. Anti-HA antibodies were used to detect a band corresponding to the predicted size for the TIP1 protein (data not shown). No bands were detected when these membranes were probed with control cell extracts or when membranes generated from BL21 crude cell extracts were probed with TGB12K-HA (data not shown). These data confirmed that protein-protein interactions occur between TGB12K and TIP1.

\section{TIP1, TIP2, and TIP3 are}

ankyrin repeat-containing proteins.

Comparison of the nucleotide sequences of each TIP with the GenBank EST databases revealed homologies with several sequences from tomato and potato. The nucleotide and translated sequences of each TIP were also compared with the GenBank nonredundant databases, using the BLASTN, BLASTX, and TBLASTX search programs (Altschul et al. 1997). These searches revealed that TIP1 is the unpublished ankyrin repeat protein HBP1 from N. tabacum (GenBank accession number AF352797; Fig. 2B). All three TIP sequences showed significant homology to two Arabidopsis thaliana proteins. These proteins are assigned as the ankyrin repeat-containing protein 2 (AKR2 or AFT) (submitted as GenBank accession numbers U70425, AF386982, and AF034387) and as a putative glucanase (GenBank accession number NM_127294; Fig. 2B). However, we were unable to identify any significant homology between this "putative glucanase" and any known glucanases. We suspect that the denomination "putative glucanase" most likely relates to the presence of a motif similar to the glycosyl hydrolase family I signature (Wang et al. 1998). The TIP sequences also showed significant homology to possible apospory-associated proteins from Pennisetum ciliare (GenBank accession number U13149) and from Oryza sativa (GenBank accession number AF358770) (Fig. 2B).

The TIP1 sequence was submitted to the FUGUE structural homology recognition server (Shi et al. 2001) to search for protein structure homologues. Five significant hits (z-scores 8.7 to 11.8) were identified, all of which contained ankyrin repeat do-

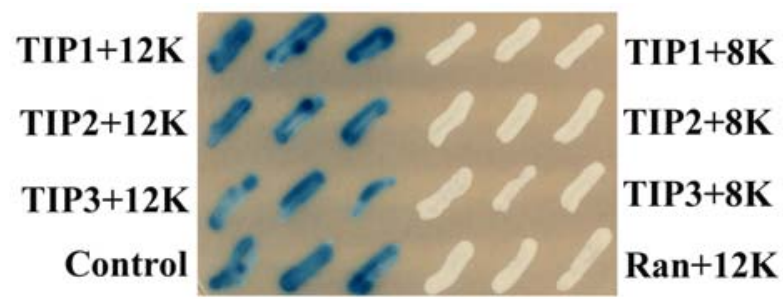

Fig. 1. Yeast two-hybrid system to identify TGB $12 \mathrm{~K}$-interacting proteins. Growth of yeast on Gal/Raf/X-Gal/SD/-His/-Trp/-Ura indicator plates. Yeast containing the reporter, bait, and prey plasmids will grow on this assay medium irrespective of whether the bait and prey proteins interact. The blue coloration indicates a protein-protein interaction between the bait construct TGB12K and the library-encoded prey proteins (TIP1, TIP2, and TIP3). White coloration indicates no interaction between the bait construct TGB8K and TIP1, TIP2, or TIP3 or between the bait construct TGB12K and the plant protein ran (similar negative interactions were observed between TGB12K and the plant proteins parf2 or protochlorophyllide reductase). Control $=$ yeast expressing the Clontech positive control plasmids pB42AD-T and pLexA-53. Three streaks of each interaction assay are shown. 
mains. The FUGUE program predicted that the ankyrin repeats were found between residues 105 and 350 of TIP1. Ankyrin repeats have been identified in a diverse group of proteins including channels, toxins, enzymes, and proteins involved in cytoskeletal organization, signal transduction, and transcription regulation. The repeats are thought to mediate protein-protein interactions (Rubtsov and Lopina 2000; Sedgwick and Smerdon 1999). A separate FUGUE search on the first 104 residues of TIP1 failed to find any significant hits.

Using the program PEST-FIND (Rechsteiner and Rogers 1996), we identified one possible PEST domain in the first 104 residues of TIP1 (HBP1) (PEST-FIND score +17.31, residues 24 to 39) (Fig. 2A). No PEST domain was identified in TIP2 or TIP3. PEST regions are rich in proline, glutamate, serine, and threonine and act as proteolytic signals targeting proteins for rapid degradation by the $26 \mathrm{~S}$ proteasome (Rechsteiner and Rogers 1996).

\section{Subcellular localization of TIP1 (HBP1).}

We decided to focus our subsequent analyses on TIP1 (HBP1). Thus, to assess the subcellular localization of TIP1, we generated a TIP1::green fluorescent protein (GFP) fusion. The TIP1::GFP fusion protein interacted with TGB12K in the yeast two-hybrid system (Table I), indicating that GFP did not affect the TGB12K binding properties of TIP1. We used a transient expression assay based on Agrobacterium tumefaciens infiltration of $N$. benthamiana leaves to introduce a $35 \mathrm{~S}$ TIP1::GFP expression cassette into plant cells.

Leaves were infiltrated with $35 \mathrm{~S}-\mathrm{TIP} 1:: \mathrm{GFP}$ to determine the subcellular location of TIP1 or with $35 \mathrm{~S}$-TIP1::GFP plus a 35S-TGB12K expression cassette to study the effect, if any, of coexpressing TIP1 with TGB12K. At 3 days postinfiltration (dpi) leaves were imaged, using confocal microscopy. Infiltration of the control construct, a 35S-GFP expression cassette, revealed green fluorescence throughout the cytoplasm and nucleus (Fig. 3A), consistent with previous reports of the cellular location for the nontargeted derivative of GFP (Haseloff et al. 1997). Leaves infiltrated with 35S-TIP1::GFP showed GFP activity similar to the pattern of fluorescence observed with free GFP (Fig. 3B), indicating that TIP1 is uniformly distributed throughout the cytoplasm. However, unlike the cells expressing free GFP, fluorescence did not accumulate in the nucleus (arrows, Fig. 3A and B).

In leaves coexpressing TIP1::GFP plus TGB12K, we observed a dramatic change in the distribution of GFP activity. In the presence of TGB12K, we observed large fluorescent bodies at the cell periphery, suggesting that TIP1::GFP was accumulating in these structures (Fig. 3C). As in cells expressing TIP1::GFP alone, no accumulation of GFP was observed in the nuclei of cells coexpressing TIP1::GFP plus TGB12K.

To confirm that accumulation of TIP1::GFP in these peripheral bodies was due to coexpression with TGB12K and not a consequence of overexpressing a foreign protein, we coexpressed TIP1::GFP with TGB8K. In these cells, fluorescent peripheral bodies were not observed, suggesting that this TGB protein does not induce the cellular changes associated with TGB12K (Fig. 3D). These data indicate that TGB12K alone or TGB12K plus TIP1 is responsible for the induction of the peripheral bodies.

To test whether these peripheral bodies also occur during virus infection, we co-expressed TIP1::GFP plus PVX in the transient expression assay. At 3 dpi, GFP activity was again associated with peripheral bodies (Fig. 3E). In leaves coexpressing PVX plus the control 35S-GFP, we also observed the fluorescent peripheral bodies (Fig. 3F). The localization of both free GFP and TIP::GFP to peripheral bodies suggests that these bodies may comprise cytoplasm. These data indicate that TGB12K can mediate the cellular changes observed at the cell periphery and that the other viral proteins do not interfere with or contribute to these changes.

To confirm the nature of the fluorescent peripheral bodies, we examined tissue sections of infiltrated leaves by electron microscopy. In leaves expressing TIP1::GFP, the cytoplasm was normal in appearance, i.e., identical to that observed in noninfiltrated cells (Fig. 4A). These results ruled out any possibility that the Agrobacterium infiltration procedure was affecting cell structure. In leaves coexpressing TIP1::GFP plus TGB12K or TIP1::GFP plus PVX or in leaves expressing PVX alone there were many distinct regions at the cell periphery where there was a change in the appearance of the cytoplasm (Fig. 4B, D, and E). The enlarged areas of cytoplasm extended out into the cell, distorting the vacuole. In PVX-infected cells (PVX alone or PVX plus TIP1::GFP), these enlarged regions of cytoplasm contained the laminate inclusion bodies characteristic of PVX infections (Shalla and Shepard 1972) (Fig. 4F, pinwheel structures). These laminate inclusion bodies were never detected in cells coexpressing TIP1::GFP plus TGB12K (Fig. 4B and C), that is, in the absence of other PVX proteins.

\section{TIP1, TIP2, and TIP3 interact with $\beta$-1, 3 -glucanase.}

To gain insight into the function of the TIPs in virus infection, we decided to identify plant proteins that might interact with them. To identify the possible targets for TIP1, we predicted that, by using this protein as bait in a screen of the $N$. tabacum cDNA yeast two-hybrid library, we would possibly identify proteins that interact with TIP1, some of which might be cell-wall associated. However, this approach was unsuccessful, because TIP1 expressed as bait activated transcription of the lac $Z$ reporter gene in yeast. That is, when expressed in the DNA-binding domain plasmid (pLexA), TIP1 activated transcription even in the absence of the activation domain plasmid (p42AD). Transcriptional activation, in the absence of pLexA, was never observed when TIP1 was expressed in $\mathrm{p} 42 \mathrm{AD}$.

As an alternative approach to identify a putative proteinbinding partner for TIP1, we chose to directly test candidate genes selected from the literature. Since callose turnover is thought to regulate plasmodesmal SEL and class I $\beta$-1,3-gluca-

Table 1. Protein-protein interactions identified using the yeast two-hybrid system ${ }^{\mathrm{a}}$

\begin{tabular}{|c|c|c|c|c|c|c|c|c|}
\hline Protein & TIP1 & TIP2 & TIP3 & TIP1::GFP & GLU & Ran & Parf2 & Protochlorophyllide reductase \\
\hline TGB25K & - & - & - & - & - & - & - & - \\
\hline TGB12K & + & + & + & + & _- & _- & _- & _- \\
\hline TGB8K & - & - & - & - & - & - & - & + \\
\hline GLU & + & + & + & + & _- & _- & _- & _- \\
\hline Ran & - & - & - & - & & & & \\
\hline Parf2 & - & - & - & - & & & & \\
\hline Protochlorophyllide reductase & _- & - & - & - & & & & \\
\hline
\end{tabular}

$\overline{\mathrm{a}}+=$ blue coloration of yeast grown on assay medium (Gal/Raf/X-Gal/SD/-His/-Trp/-Ura) indicating a positive protein-protein interaction; and $-=$ white coloration on assay medium indicating no protein interaction. 
nase has been implicated in facilitating virus movement by degrading callose (Beffa et al. 1996; Bucher et al. 2001; Iglesias and Meins 2000), $\beta$-1,3-glucanase was a possible candidate for a TIP-interacting protein.

We investigated whether TIP1, TIP2, and TIP3 would interact directly with a $\beta$-1,3-glucanase (GLU) from $N$. tabacum (GenBank accession number M60402) by testing for an interaction in yeast. For this, we mated yeast strains carrying p42AD-TIP1 with cells carrying pLexA-GLU. Plasmid p42AD-TIP1 was also introduced into yeast expressing LexA fused to one of three unrelated plant proteins as controls (ran, parf2, and protochlorophyllide reductase). The two-hybrid assay indicated that the reporter genes were activated in yeast expressing both TIP1 and GLU (Fig. 5, Table 1) but not in cells expressing TIP1 plus the control proteins (Table 1). Likewise, TIP2 and TIP3 interacted with GLU (Fig. 5, Table 1) but not with the control proteins (Table 1), consistent with the idea that all three TIPs may have similar functions. GLU interacted with TIP1::GFP but not with TGB25K, TGB12K, or TGB8K (Fig. 5, Table 1) nor with the control plant proteins (Table 1). Our two-hybrid data suggest that specific protein-protein interactions occur between TGB12K and all three TIPs and between GLU and all three TIPs but not between GLU and TGB12K.

The specific interaction between GLU and the TIPs was confirmed by far-Western analysis, as described above. We expressed GLU tagged with the HA peptide sequence (YPYDVPDYA) in E. coli strain BL21. The membrane-bound target proteins (crude protein extract from BL21 expressing TIP1) were probed with crude cell extract of BL21 expressing GLU-HA. Anti-HA antibodies were used to detect a band corresponding to the predicted size for the TIP1 protein (data not shown). No bands were detected when these membranes were probed with control cell extracts or when membranes generated from BL21 crude cell extracts were probed with GLU-HA (data not shown). These data confirmed that protein-protein interactions occur between GLU and TIP1.

\section{DISCUSSION}

During PVX infection of host plants, there is an absolute requirement for TGB12K to increase the plasmodesmal SEL (Tamai and Meshi 2001). To further our understanding of the mechanism by which this MP regulates plasmodesmata, we searched for plant proteins that interact directly with TGB12K. We identified three very similar proteins (termed here as TIP1, TIP2, and TIP3) that specifically interact with TGB12K.

We propose that induction of the TIPs is not dependent on virus infection. We conclude this because TIP cDNAs were detected in noninfected leaves and shoots (based on their appearance in the yeast two-hybrid library and on our RT-PCR studies). Also, we were unable to detect any change in TIP expression when we compared healthy leaves with PVXinfected samples, suggesting that TIP expression is not altered by the virus.
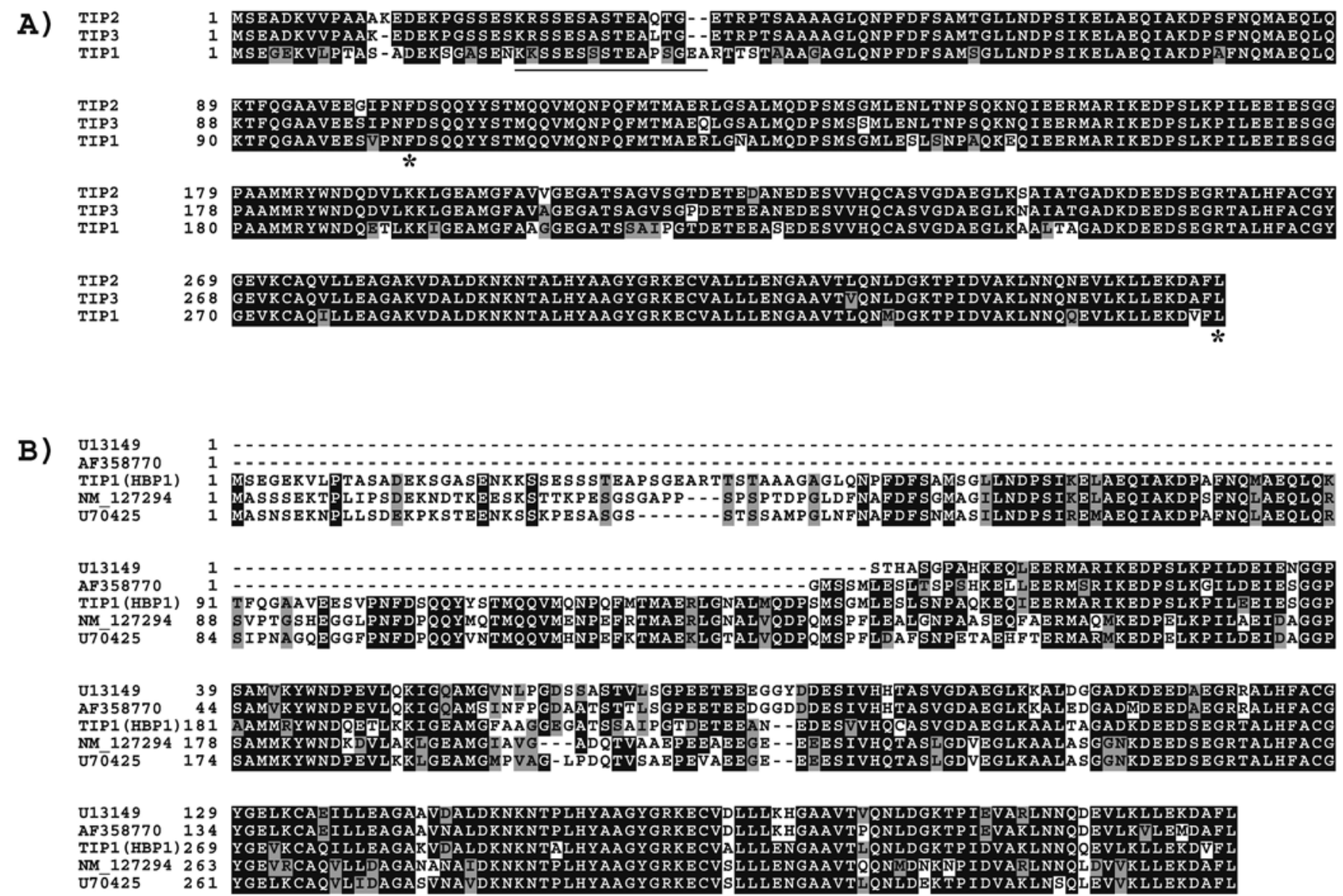

Fig. 2. Amino acid sequence alignments. A, Alignment of TIP1, TIP2, and TIP3 deduced protein sequences. The PEST domain in TIP1 is located at residues 24 to 39 and is marked by a horizontal line below the TIP1 sequence. The ankyrin repeats domains are found between residues 105 to 350 . The region containing these domains is delineated by asterices. B, Alignment of TIP1 (HBP1) (Nicotiana tabacum)-deduced protein sequence with translated sequences from the GenBank databases: U13149, possible apospory-associated protein (Pennisetum ciliare); AF358770, apospory-associated protein (Oryza sativa); NM_127294, "putative glucanase" (Arabidopsis thaliana); and U70425, the ankyrin repeat-containing protein AKR2 (A. thaliana). The alignment of the apospory-associated proteins suggest that the database sequences are in a truncated form. Regions of identity and similarity are indicated by black and grey boxes, respectively. Gaps introduced for alignment are indicated by dashes. 
Our subcellular localization studies revealed that TIP1::GFP accumulates in the cytoplasm. In the presence of TGB12K, this fusion protein localized to deposits of additional cytoplasm. Solovyev and associates (2000) showed that PVX TGB12K::GFP was associated with peripheral bodies but only in the presence of TGB8K. Here, we have shown that none of the other PVX proteins, including TGB8K, are required to induce the formation of cytoplasmic deposits to which the TIP1::GFP fusion protein localizes.

We propose that TGB $12 \mathrm{~K}$ mediates the deposition of additional cytoplasm at the cell periphery. This additional cytoplasm accommodates the inclusion bodies that are characteristic of PVX infections (Davies et al. 1993; Shalla and Shepard 1972). TGB25K and TGB12K (in the presence of TGB8K) were shown to localize to peripheral bodies (Davies et al. 1993; Solovyev et al. 2000). Furthermore, the Benyvirus P51 protein (the PVX TGB25K counterpart) was shown to accumulate in peripheral bodies (Erhardt et al. 2000). More importantly, these peripheral bodies colocalized with callose (Erhardt et al. 2000). Thus, we propose that these bodies represent sites where the virus transport complex may interact with cellular components to bring about the plasmodesmatal modifications required to allow passage of the virus between cells.

For PVX to undergo cell-to-cell movement, previous work has implicated both GLU as a regulator of plasmodesmal SEL through callose degradation (Iglesias and Meins 2000) and TGB12K as the viral protein responsible for mediating the increase in the SEL (Tamai and Meshi 2001). Here, we have identified the host factor that links these two key components involved in PVX movement. We propose that increased callose deposition in the neck region of plasmodesmata occurs in response to PVX infection and that TGB12K increases the plasmodesmal SEL via callose degradation. Our data suggest that TGB12K mediates this effect via TIP and GLU. Because GLU can interact directly with the TIPs in the absence of TGB12K, it seems unlikely that TGB $12 \mathrm{~K}$ induces a conformational change in TIP that promotes its interaction with GLU. A more plausible explanation is that TGB $12 \mathrm{~K}$ targets TIP to GLU. Alternatively, TGB12K may translocate GLU from the vacuole, in which it is normally expressed (Van den Bulcke et al. 1989), to the cell wall via an interaction with TIP. GLU is induced during pathogen attack (Linthorst 1991), and its main function in a fungal infection is to degrade the fungal cell walls. Thus, in our model, we propose that PVX exploits a host defense response to promote its cell-to-cell spread and that the virus achieves this via TGB12K-TIP and TIP-GLU interactions.

The plasmodesmata in PVX-infected leaves appear to have also lost the desmotubule and are distorted in shape (Santa Cruz et al. 1998). Therefore, it is unlikely that opening of the neck region through callose degradation is the only modification to plasmodesmata that occurs during viral movement. Understanding how the PVX proteins or the host factors, or both, mediate this apparent rearrangement of the internal components of plasmodesmata remains a further challenge.

Since different viruses have evolved different mechanisms for movement, it is probable that they interact with different host factors. It will be interesting to determine whether the MPs of Tobacco mosaic virus (TMV) and Cucumber mosaic virus (CMV)
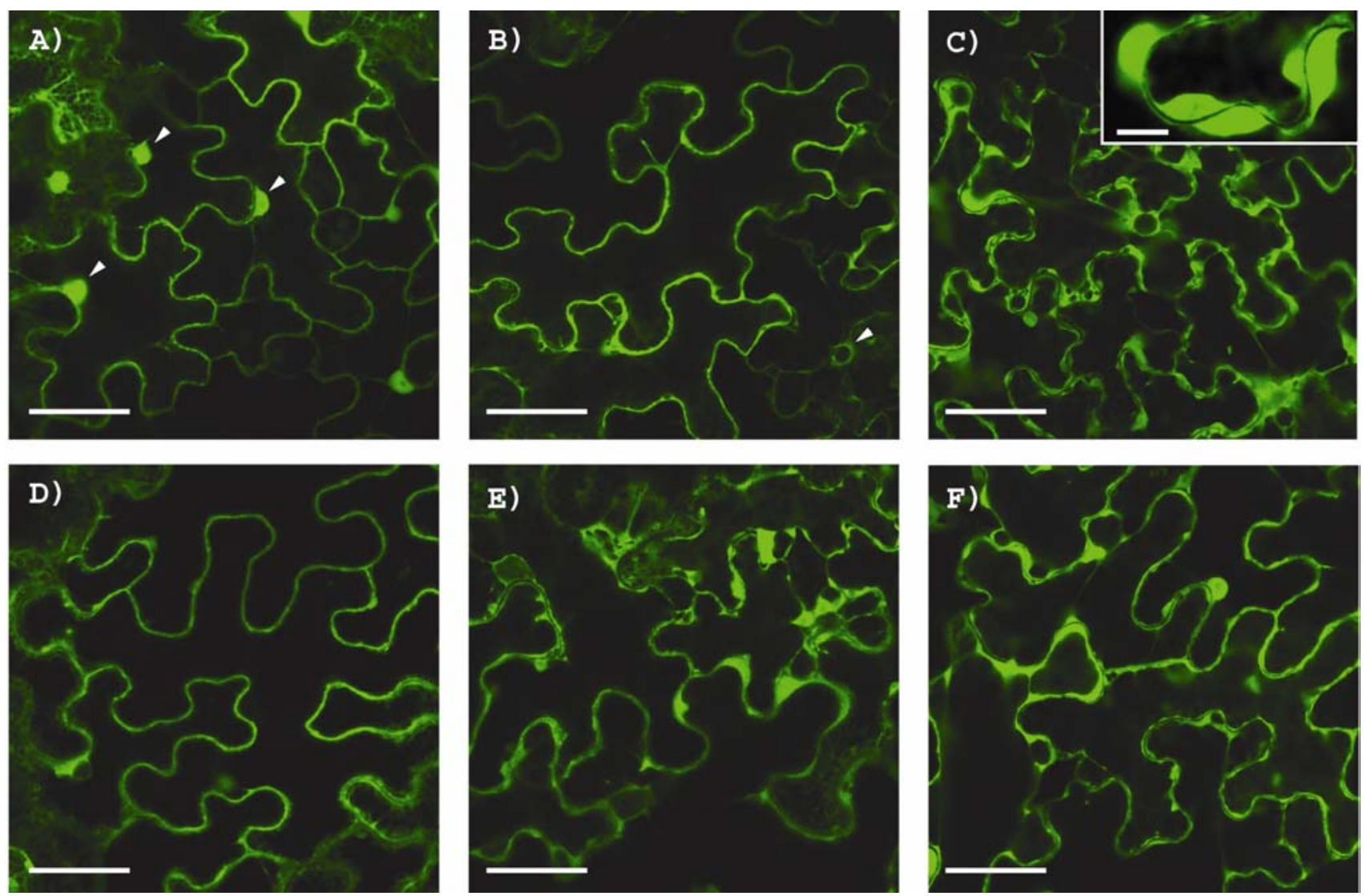

Fig. 3. Fluorescence microscopy of Nicotiana benthamiana leaves transiently expressing green fluorescent protein (GFP) and TIP1::GFP fusion proteins. Agrobacterium tumefaciens-infiltrated leaves were imaged at 3 days postinoculation using confocal microscopy. A, Fluorescence of free GFP; arrows point to nuclei. B, Fluorescence of TIP1::GFP fusion protein; the arrow points to a nucleus. C, Localization of fluorescence in peripheral bodies in cells coexpressing TIP1::GFP and TGB12K. Inset shows a higher magnification of the peripheral bodies. D, Fluorescence of TIP1::GFP in cells coexpressing the fusion protein and TGB $8 \mathrm{~K}$. E, Localization of fluorescence in peripheral bodies in cells coexpressing TIP1::GFP and Potato virus $X$ (PVX). F, Localization of fluorescence in cells coexpressing PVX and free GFP. Scale bars in all panels represent $50 \mu \mathrm{m}$, except the inset in panel C that represents $10 \mu \mathrm{m}$. A representative set of images is shown. 
also interact with the TIPs or with other TIP-like factors, since it has been proposed that these viruses, like PVX, also increase plasmodesmal SEL through regulating callose turnover (Bucher et al. 2001; Iglesias and Meins 2000). Pectin methylesterase and 2b-binding protein have been shown to interact with the MPs of TMV and CMV, respectively, but neither are TIP-like proteins nor have they been implicated in callose turnover (Chen et al. 2000; Dorokhov et al. 1999; Ham et al. 1999).

Peck and associates (2001) showed that one of the $A$. thaliana TIP homologues (the "putative glucanase" designated AtPhos 43 by these authors) is rapidly phosphorylated in response to fungal and bacterial elicitor treatment. Phosphorylation plays a key role in defense signaling-proteins that are rapidly phosphorylated during pathogen attack are often components of signal transduction pathways. This finding raises the interesting possibility that the TIPs are also phosphorylated during PVX infection. If they are, we will need to determine the significance of this in relation to the plant's perception and response to pathogen attack.

The other A. thaliana TIP homologue, the AKR2 (AFT) protein, was recently identified in a yeast two-hybrid screen using a 14-3-3 protein as bait (Yan et al. 2002). Down-regulation of this gene in A. thaliana, using antisense technology, resulted in plants exhibiting altered phenotypes. These silenced plants showed increased PR-1 gene expression, increased expression of glutathione S-transferase 6, and increased resistance to bacterial infection (Yan et al. 2002). These authors concluded that AKR2 may play an important role in regulating disease and stress responses. Thus, both A. thaliana TIP homologues have been implicated in disease responses.

A major challenge for the future will be to elucidate the roles of the TIPs in healthy plants. Expression of the TIPs in nonin-
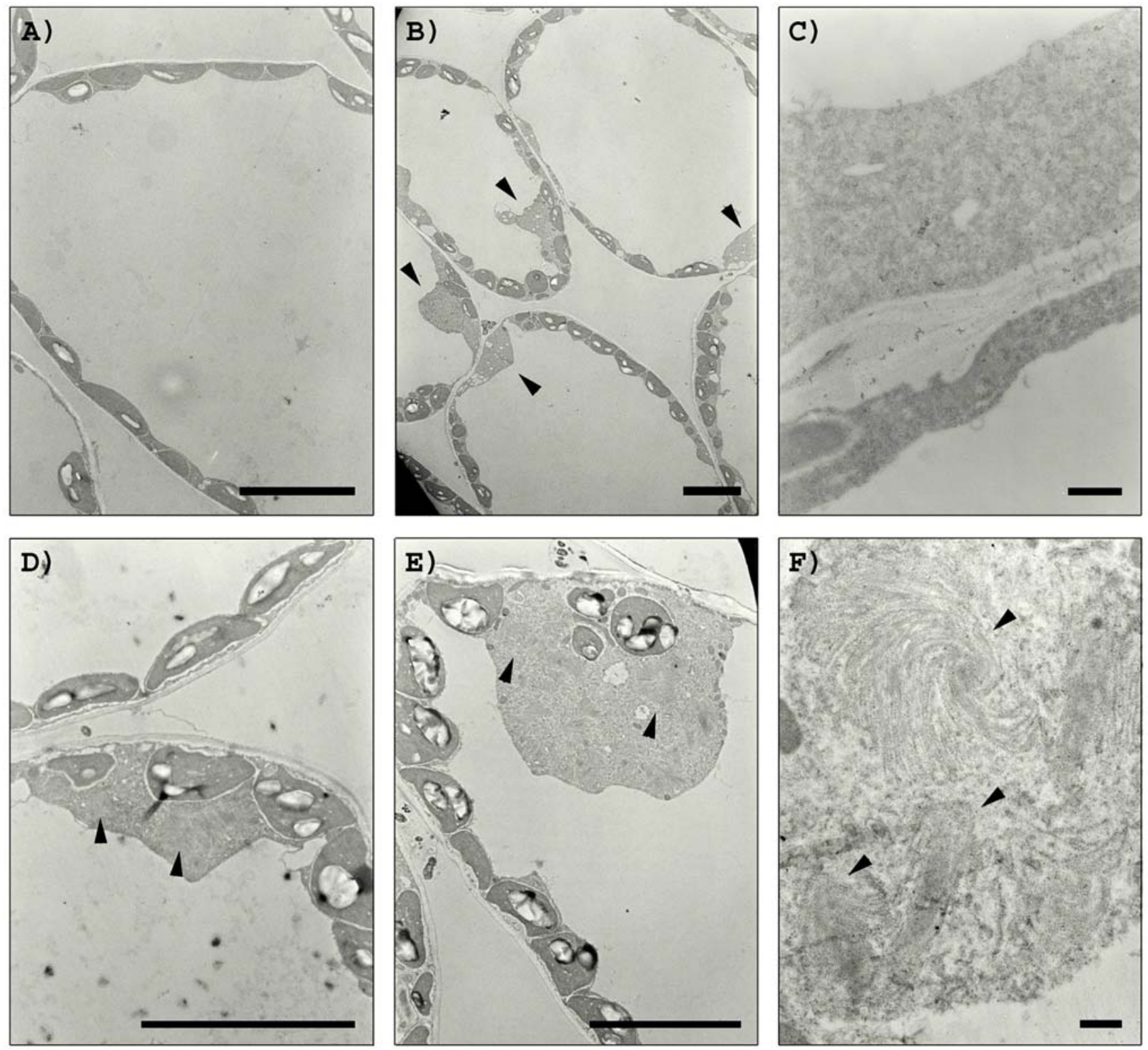

Fig. 4. Micrographs of cells transiently expressing TIP1::GFP and TGB12K. Sections were taken from Nicotiana benthamiana leaves transiently expressing constructs at 3 days postinoculation. A representative set of sections are shown. A, A cell expressing TIP1::GFP showing cytoplasm of normal appearance. B, Cells coexpressing TIP1::GFP plus TGB12K reveal cellular changes, as indicated by the deposition of additional cytoplasm, indicated by arrows. C, A higher magnification view on one of the areas of enlarged cytoplasm from panel B showing adjacent cells. The cytoplasm does not appear to contain the inclusion bodies that are present in cells infected with Potato virus X (PVX) (panels D, E, and F). D, Additional cytoplasm is deposited in cells infected with PVX and contain inclusion bodies characteristic of PVX infections (arrows). E, Cell coexpressing PVX and TIP1::GFP shows deposition of additional cytoplasm containing laminate inclusion bodies (arrows). F, Higher magnification of a laminate inclusion body from panel E showing pinwheel structures (arrows). In A, B, D, and E, the scale bar represents $10 \mu \mathrm{m}$; in panels $\mathrm{C}$ and F, $500 \mathrm{~nm}$. 
fected plants suggests that these host proteins may be part of the endogenous intercellular trafficking system, regulating the movement of macromolecules between cells. Perhaps the TIPs also interact with plant proteins in response to stimuli other than TGB12K. If the TIPs identified here represent three members of a highly conserved gene family, then it is tempting to speculate that different TIP-like proteins may respond to different signals to regulate GLU activity and, hence, macromolecular trafficking.

\section{MATERIALS AND METHODS}

\section{Yeast library transformation, screening, and matings.}

Total RNA was extracted from four- to five-week-old noninfected N. tabacum cv. Samsun NN plants. PolyA RNA was isolated using oligo $\mathrm{d}(\mathrm{T})$ magnetic beads and first-strand cDNA was synthesized using a primer incorporating an XhoI site for cloning. cDNA was synthesized using the Stratagene cDNA synthesis kit (Stratagene, La Jolla, CA, U.S.A.). Adapter sequences containing EcoRI sites were ligated to the doublestranded cDNA. After digestion with EcoRI and XhoI, the cDNAs were ligated to the similarly digested Clontech LexAbased yeast two-hybrid vector pB42AD (Clontech, Palo Alto, CA, U.S.A.). Two libraries were prepared with average insert sizes of 1.4 (library 1) and $0.9 \mathrm{~kb}$ (library 2). The number of clones in the primary libraries were $0.5 \times 10^{6}$ and $1.8 \times 10^{6}$ for library 1 and library 2 , respectively.

The open reading frame (ORF) of PVX TGB12K was synthesized by PCR, using pTXS (the full-length cDNA clone of $\left.\mathrm{PVX}_{\mathrm{UK} 3}\right)$ as template and primers incorporating EcoRI and XhoI sites at the start and end of TGB $12 \mathrm{~K}$, respectively. The TGB12K was cloned as a translational fusion with the LexA binding domain in pLexA to generate the bait plasmid, LexATGB12K. Yeast strain EGY48(p8op-lacZ) was transformed with LexA-TGB12K.

Libraries 1 and 2 (33 and $5 \mu \mathrm{g}$, respectively) were pooled and transformed into yeast strain EGY48(p8op-lacZ) carrying the LexA-TGB12K bait plasmid. The amplified yeast library was titred, and cotransformants were plated at high density (about $10^{6}$ $\mathrm{CFU}$ per plate) on assay medium (SD/Gal/Raf/-His/-Leu/-Trp/$\mathrm{Ura} / \mathrm{X}$-gal). Plates were incubated at $28^{\circ} \mathrm{C}$ and, four days after plating, were scored for the appearance of blue colonies.

For mating experiments, the PVX TGB8K ORF was synthesized by PCR using pTXS and primers incorporating EcoRI and $\mathrm{XhoI}$ sites at the start and end of TGB8K, respectively. The TGB8K was cloned as a translational fusion with the LexA binding domain in pLexA to generate the bait plasmid, LexA-

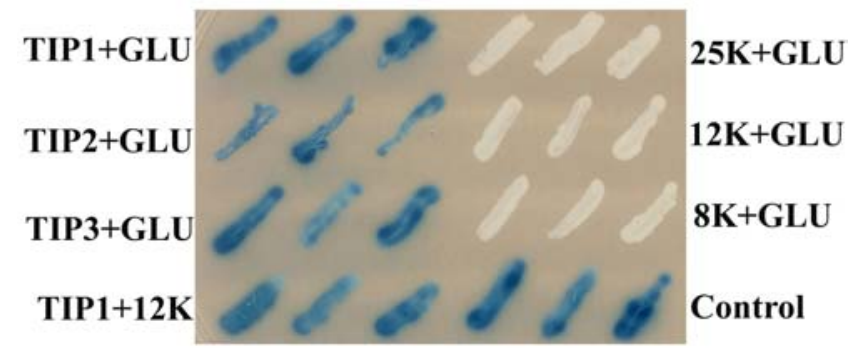

Fig. 5. Yeast two-hybrid system to identify TIP-interacting proteins. Growth of yeast on Gal/Raf/X-Gal/SD/-His/-Trp/-Ura indicator plates. This medium will allow growth of yeast containing the reporter, bait, and prey plasmids irrespective of whether the bait and prey proteins interact. The blue coloration indicates protein-protein interactions between the TIPs and $\beta$-1,3-glucanase (GLU). White coloration indicates no interaction between GLU and TGB12K nor between GLU and TGB8K or TGB25K. TIP1 plus TGB12K represents the positive control for interaction. Control = yeast expressing the Clontech positive control plasmids pB42AD-T and pLexA53. Three streaks of each interaction assay are shown.
TGB8K. Control constructs were produced expressing one of three plant proteins (tobacco Ran, Parf2, or the tobacco homologue of protochlorophyllide reductase from tomato). The fulllength tobacco ran and parf2 cDNA sequences (provided by B. Drøbak) and the protochlorophyllide reductase cDNA sequence (GenBank accession number AF243522) were synthesized by PCR, using primers incorporating EcoRI and XhoI sites at the start and end of each cDNA, respectively. These sequences were then introduced into pLexA. The TIP1, TIP2, and TIP3 sequences were directly transferred from the prey plasmid (p42AD) into the bait plasmid (pLexA) by EcoRI-XhoI digestion. These pLexA-based clones always activated transcription of the reporter genes in the absence of the activation domain plasmid (p42AD) and were, therefore, unsuitable in further studies. In yeast matings, the TIP clones were always expressed from the p42AD vector. TGB12K and TGB8K were directly transferred from the bait plasmid (pLexA) into the prey plasmid (p42AD) by EcoRI-XhoI digestion. The GLU ORF (GenBank accession number M60402) was synthesized by PCR, using primers incorporating cloning sites from $N$. $t a$ bacum cv. Samsun NN cDNA. After digestion with EcoRI and XhoI, the GLU fragment was cloned into both pLexA and p42AD.

Yeast strains EGY48(p8op-lacZ) and YM4271 were transformed with all constructs. The Clontech pLexA-53 and p42AD$\mathrm{T}$ plasmids were used as a control for a positive protein-protein interaction. To identify interactions after mating experiments, assay medium was SD/Gal/Raf/-His/-Trp/-Ura/X-gal. This medium allowed growth of all yeast-containing prey, bait, and reporter plasmids irrespective of whether the bait and prey proteins interacted. Positive interactions were scored as blue coloration of yeast. All clones were verified by sequencing. All yeast medium, yeast transformations, and matings were as described in the Clontech LexA two-hybrid instruction manual.

\section{Construction of expression cassettes}

for confocal and transmission microscopy.

A 35S-GFP-nopaline synthase (nos) terminator expression cassette was introduced into the EcoRI and HindIII sites of the pBIN19 binary vector. The GFP derivative used was a nontargeted form of GFP, provided by J. Haseloff. For the TIP1::GFP fusion protein construct, full-length TIP1 was generated by PCR and was cloned as a translational fusion with GFP between the $35 \mathrm{~S}$ promoter and the nos terminator to generate 35S-TIP1::GFP-nos. This expression cassette was transferred into pBIN19 as an EcoRI-HindIII fragment. For coexpression of TGB12K with GFP, a 35S-TGB12K-ocs cassette was cloned into the HindIII site of pBIN19 carrying the expression cassette 35S-GFP-nos. Similarly, for coexpression of TGB12K and TIP1::GFP, a 35S-TGB12K-ocs cassette was cloned into the HindIII site of pBIN19 carrying the 35S-TIP1::GFP-nos cassette. For coexpression of TGB8K and TIP1::GFP, a 35STGB8K-ocs cassette was cloned into the EcoRI site of pBIN19 carrying the 35S-TIP1::GFP-nos construct. The full-length PVX clone expressed from the $35 \mathrm{~S}$ promoter (in a pGREEN binary vector) was provided by $\mathrm{D}$. Baulcombe. All constructs were verified by sequencing.

The plasmids were introduced into A. tumefaciens strain $\mathrm{C} 58 \mathrm{C} 1$ (pCH32) using electroporation at 400 omega and 2500 $\mathrm{V}$ in a gene pulser apparatus (Bio-Rad, Hercules, CA, U.S.A.). Transformants were selected on L-agar supplemented with 50 $\mu \mathrm{g}$ of kanamycin per $\mathrm{ml}$ and $3 \mu \mathrm{g}$ of tetracycline per $\mathrm{ml}$.

\section{Transient expression assays} and $A$. tumefaciens infiltrations.

A. tumefaciens strain $\mathrm{C} 58 \mathrm{C} 1$ ( $\mathrm{pCH} 32)$ containing individual binary vectors was inoculated to $10 \mathrm{ml}$ L-broth supplemented 
with $50 \mu \mathrm{g}$ of kanamycin per $\mathrm{ml}$ and $3 \mu \mathrm{g}$ of tetracycline per $\mathrm{ml}$ and grown at $28^{\circ} \mathrm{C}$ for 2 days. This culture $(1 \mathrm{ml})$ was inoculated into $50 \mathrm{ml}$ L-broth supplemented with $10 \mu \mathrm{l}$ of $100 \mathrm{mM}$ acetosyringone plus kanamycin and tetracycline. After overnight incubation at $28^{\circ} \mathrm{C}$, cells were collected by centrifugation, were resuspended in $30 \mathrm{ml}$ of $10 \mathrm{mM} \mathrm{MgCl}_{2}$, supplemented with $5 \mu$ of 100 $\mathrm{mM}$ acetosyringone, and were incubated at room temperature for more than $1 \mathrm{~h}$.

A. tumefaciens infiltration was conducted on expanding leaves of $N$. benthamiana plants. For coexpression of PVX plus TIP1::GFP or PVX plus free GFP, individual cultures were mixed in a ratio of $1: 1$ prior to infiltration. Leaf samples for confocal or transmission electron microscopy were collected at 3 dpi. For all A. tumefaciens infiltration experiments, a set of noninfiltrated plants was also used as control.

\section{Imaging of GFP.}

A. tumefaciens-infiltrated leaves were detached at 3 dpi and were viewed with a Leica DMR confocal laser scanning microscope (Leica, Behsheim, Germany) and a 40× oil objective to image GFP fluorescence. The excitation wavelength was 488 $\mathrm{nm}$ using an Argon laser, and a filter of 500 to $545 \mathrm{~nm}$ was used for emission.

\section{Transmission electron microscopy.}

The tobacco leaf samples were cut into $1 \mathrm{~mm}^{3}$ pieces and immediately placed in $2.5 \%$ ( vol/vol) glutaraldehyde in $0.05 \mathrm{M}$ sodium cacodylate, $\mathrm{pH} 7.3$, overnight at room temperature to fix the tissue. The samples were placed in tissue-handling devices and were processed at low temperature as described by Wells (1985).

\section{ACKNOWLEDGMENTS}

We thank M. Durrant for the protein structure analyses, G. Lomonossoff, J. Stanley, and A. Maule for their critical reading of the manuscript and for many helpful discussions, S. Gardner and C. Thomas for assistance and technical advice with the yeast two-hybrid screens, G. Calder for technical assistance with confocal microscopy, J. Haseloff for providing 35S-GFP, B. Drøbak for providing the tobacco Ran and Parf2 cDNAs, and D. Baulcombe for providing PVX. I. F. was in receipt of a European Molecular Biology Organization long-term fellowship. J. G. was supported by a Biotechnology and Biological Sciences Research Council (BBSRC)/ National Environment Research Council (NERC) gene flow in plants and microorganisms initiative. The John Innes Centre receives a Core Strategic Grant from the BBSRC.

\section{LITERATURE CITED}

Altschul, S. F., Madden, T. L., Schäffer, A. A., Zhang, J. H., Zhang, Z., Miller, W., and Lippman, D. J. 1997. Gapped BLAST and PSIBLAST: A new generation of protein database search programs. Nucleic Acids Res. 25:3389-3402.

Angell, S. M., Davies, C., and Baulcombe, D. C. 1996. Cell-to-cell movement of potato virus $\mathrm{X}$ is associated with a change in the sizeexclusion limit of plasmodesmata in trichome cells of Nicotiana clevelandii. Virology 216:197-201.

Beffa, R. S., and Meins, F. 1996. Pathogenesis-related functions of plant $\beta$-1,3-glucanases investigated by antisense transformation-a review. Gene 179:97-103.

Botha, C. E. J., and Cross, R. H. M. 2000. Towards reconciliation of structure with function in plasmodesmata-who is the gatekeeper? Micron. 31:713-721.

Bucher, G. L., Tarina, C., Heinlein, M., Di Serio, F., Meins, F., and Iglesias, V. A. 2001. Local expression of enzymatically active class I $\beta$-1,3-glucanase enhances symptoms of TMV infection in tobacco. Plant J. 28:361-369.

Carrington, J. C., Kasschau, K. D., Mahajan, S. K., and Schaad, M. C. 1996. Cell-to-cell and long-distance transport of viruses in plants. Plant Cell 8:1669-1681.

Chapman, S., Kavanagh, T., and Baulcombe, D. C. 1992. Potato virus X as a vector for gene expression in plants. Plant J. 2:549-557.
Chen, M.-H., Sheng, J., Hind, G., Handa, A. K., and Citovsky, V. 2000 Interaction between the tobacco mosaic virus movement protein and host cell pectin methylesterases is required for viral cell-to-cell movement. EMBO (Eur. Mol. Biol. Organ.) J. 19:913-920.

Davies, C., Hills, G., and Baulcombe, D. C. 1993. Sub-cellular localisation of the $25-\mathrm{kDa}$ protein encoded in the triple gene block of potato virus X. Virology 197:166-175.

Derrick, P. M., Barker, H., and Oparka, K. 1992. Increase in plasmodesmatal permeability during cell-to-cell spread of tobacco rattle virus from individually inoculated cells. Plant Cell 4:1405-1412.

Dorokhov, Y. L., Makinen, K., Frolova, O. Y., Merits, A., Saarinen, J., Kalkkinen, N., Atabekov, J. G., and Saarma, M. 1999. A novel function for a ubiquitous plant enzyme pectin methylesterase: The host-cell receptor for the tobacco mosaic virus movement protein. FEBS (Fed. Eur. Biochem. Soc.) Lett. 461:223-228.

Erhardt, M., Morant, M., Ritzenthaler, C., Stussi-Garaud, C., Guilley, H., Richards, K., Jonard, G., Bouzoubaa, S., and Gilmer, D. 2000. P42 movement protein of Beet necrotic yellow vein virus is targeted by the movement proteins P13 and P15 to punctate bodies associated with plasmodesmata. Mol. Plant-Microbe Interact. 13:520-528.

Fedorkin, O. N., Solovyev, A. G., Yelina, N. E., Zamyatnin, A. A., Zinovkin, R. A., Makinen, K., Schiemann, J., and Yu Morozov, S. 2001. Cell-to-cell movement of potato virus $X$ involves distinct functions of the coat protein. J. Gen. Virol. 82:449-458.

Fujiwara, T., Giesman-Cookmeyer, D., Ding, B., Lommel, S. A., and Lucas, W. J. 1993. Cell-to-cell trafficking of macromolecules through plasmodesmata potentiated by the red clover necrotic mosaic virus movement protein. Plant Cell 5:1783-1794.

Haseloff, J., Siemering, K. R., Prasher, D. C., and Hodge, S. 1997. Removal of a cryptic intron and subcellular localization of green fluorescent protein are required to mark transgenic Arabidopsis plants brightly. Proc. Natl. Acad. Sci. U.S.A. 94:2122-2127.

Ham, B. K., Lee, T. H., You, J. S., Nam, Y. M., Kim, J. K., and Paek, K. H. 1999. Isolation of a putative tobacco host factor interacting with cucumber mosaic virus-encoded $2 \mathrm{~b}$ protein by yeast two-hybrid screening. Mol. Cell 9:548-555.

Iglesias, V. A., and Meins, F. 2000. Movement of plant viruses is delayed in a $\beta$-1,3-glucanase-deficient mutant showing a reduced plasmodesmal size exclusion limit and enhanced callose deposition. Plant J. 21:157-166.

Linthorst, H. J. M. 1991. Pathogenesis-related proteins of plants. Crit. Rev. Plant Sci. 10:123-150.

Lucas, W. J., and Gilbertson, R. L. 1994. Plasmodesmata in relation to viral movement within leaf tissues. Annu. Rev. Phytopathol. 32:387-411.

Northcote, D. H., Davey, R., and Lay, J. 1989. Use of antisera to localize callose, xylan and arabinogalactan in the cell-plate, primary and secondary cell walls of plant cells. Planta. 178:353 366.

Oparka, K., Roberts, A. G., Roberts, I. M., Prior, D. A. M., and Santa Cruz, S. 1996. Viral coat protein is targeted to, but does not gate, plasmodesmata during cell-to-cell movement of potato virus X. Plant J. 10:805-813.

Overall, R. L., and Blackman, L. M. 1996. A model of the macromolecular structure of plasmodesmata. Trends Plant Sci. 1:307-311.

Peck, S. C., Nuhse, T. S., Hess, D., Iglesias A., Meins, F., and Boller, T. 2001. Directed proteomics identifies a plant-specific protein rapidly phosphorylated in response to bacterial and fungal elicitors. Plant Cell 13:1467-1475.

Rechsteiner, M., and Rogers, S. W. 1996. PEST sequences and regulation by proteolysis. TIBS 21:267-271.

Rubtsov, A. M., and Lopina, O. D. 2000. Ankyrins. FEBS (Fed. Eur. Biochem. Soc.) Lett. 482:1-5.

Santa Cruz, S., Roberts, A. G., Prior, D. A. M., Chapman, S., and Oparka, K. 1998. Cell-to-cell and phloem-mediated transport of potato virus X: The role of virions. Plant Cell 10:495-510.

Sedgwick, S. G., and Smerdon, S. J. 1999. The ankyrin repeat: A diversity of interactions on a common structural framework. Trends Biochem. Sci. 24:311-316.

Shalla, T. A., and Shepard, J. F. 1972. The structure and antigenic analysis of amorphous inclusion bodies induced by potato virus $\mathrm{X}$. Virology 49:654-667.

Shi, J., Blundell, T. L., and Mizuguchi, K. 2001. FUGUE: Sequencestructure homology recognition using environment-specific substitution tables and structure-dependent gap penalties. J. Mol. Biol. 310:243-257.

Solovyev, A. G., Stroganova, T. A., Zamyatnin, A. A., Fedorkin, O. N., Schiemann, J., and Yu Morozov, S. 2000. Subcellular sorting of small membrane-associated triple gene block proteins: TGBp3-assisted targeting of TGBp2. Virology 269:113-127.

Tamai, A., and Meshi, T. 2001. Cell-to-cell movement of Potato virus X: The role of p12 and p 8 encoded by the second and third open reading 
frames of the triple gene block. Mol. Plant-Microbe Interact. 14:11581167.

Van den Bulcke, M., Bauw, G., Castresana, C., van Montagu, M., and Vandekerckhove, J. 1989. Characterisation of vacuolar and extracellular $\beta$-1,3-glucanases of tobacco: Evidence for a strictly compartmentalised plant defence system. Proc. Natl. Acad. Sci. U.S.A. 86:2673-2677.

Vaquero, C., Turner, A. P., Demangeat, G., Sanz, A., Serra, M. T., Roberts, K., and Garcia-Luque, I. 1994. The 3a protein from cucumber mosaic virus increases the gating capacity of plasmodesmata in transgenic tobacco plants. J. Gen. Virol. 75:3193-3197.

Voinnet, O., Lederer, C., and Baulcombe, D. C. 2000. A viral movement protein prevents spread of the gene silencing signal in Nicotiana benthamiana. Cell 103:157-167.

Wang, J., Hocart, C. H., Wei K.-J., and John, P. C. L. 1998. Nucleotide sequence of a cDNA clone containing tandem ankyrin repeats and a glycosyl hydrolase family I signature from Arabidopsis thaliana (accession no. AF034387). (PGR98-150) Plant Physiol. 117:1526

Wells, B. 1985. Low temperature box and tissue handling device for embedding biological tissue for immunostaining in electron microscopy. Micron and Microscopica Acta. 16:49-53.

Wolf, S., Deom, C. M., Beachy, R. N., and Lucas, W. J. 1989. Movement protein of tobacco mosaic virus modifies plasmodesmatal size exclusion limit. Science 246:377-379.

Yan, J., Wang, J., and Zhang, H. 2002. An ankyrin repeat-containing protein plays a role in both disease resistance and antioxidation metabolism. Plant J. 29:193-202.

Yang, Y., Ding, B., Baulcombe, D. C., and Verchot, J. 2000. Cell-to-cell movement of the $25 \mathrm{~K}$ protein of Potato virus $X$ is regulated by three other viral proteins. Mol. Plant-Microbe Interact. 13:599-605.

Zambryski, P., and Crawford, K. 2000. Plasmodesmata: Gatekeepers for cell-to-cell transport of developmental signals in plants. Annu. Rev. Cell Dev. Biol. 16:393-421. 